\title{
Lateral boundary conditions for a local anisotropic ice-flow model
}

\author{
Olivier GAGLIARDINI, JAGQues MEYSSONNIER \\ Laboratoire de Glaciologie et Géophysique de l'Environnement, Domaine Universitaire, 54 rue Molière, BP 96, \\ 38402 Saint-Martin-d'Hères Cedex, France \\ E-mail: gagliardini@lgge.obs.ujf-grenoble.fr
}

\begin{abstract}
A local two-dimensional flow model which accounts for the anisotropic behaviour of polar ice and the evolution of its strain-induced anisotropy is briefly reviewed. Due to its complexity, it is not yet possible to use this model to simulate the flow of a whole ice sheet, and its potential applications are presently restricted to limited spatial domains around existing drilling sites. In order to calculate the local flow of ice, boundary conditions must be applied on the lateral edges of the studied domain. Since these limits correspond to fictitious sections of the ice sheet, the type of boundary condition to adopt is not obvious. In the present paper, different kinds of boundary conditions of the Dirichlet type, applied at the lateral boundary of an idealized ice sheet of simplified geometry, are discussed. This will serve as a first step towards the coupling of the local flow model with a global ice-sheet flow model.
\end{abstract}

\section{INTRODUCTION}

In the framework of the interpretation of climatic and atmospheric ice-core records, a better understanding of the flow of ice in the vicinity of potential and existing drilling sites is required. To this end, many glaciological measurements, such as surface and bedrock topographies and surface velocities, are performed at these locations, and borehole surveys are carried out whenever possible. The spatial resolution of these measurements is very high compared to that of results derived from a global ice-sheet flow model which is relatively poor (Savvin and others, 2000). Therefore, in order to achieve a convenient accuracy, a local flow model which enables the solution of the full stress-equilibrium equations while using high-resolution topographic data, especially close to summits and ice divides where the shallow-ice approximation (SIA) is not valid, must be used. On the other hand, such a local flow model can be sought to consider a better description of the behaviour of polycrystalline ice than that used in current global ice-sheet models, in particular as regards the strong anisotropy of polar ice and the evolution of its fabric. Since, by definition, a local model simulates the flow of ice in a limited domain, the domain margins must be submitted to boundary conditions which reproduce the action of the rest of the ice sheet. When the local model is simply a higher-resolution version of the global model, i.e. when both are solving the same equations on a subgrid and a coarse grid, respectively, the simplest way to apply the boundary conditions in the local model is to interpolate the coarse-grid results at the margin subgrid points (Savvin and others, 2000). Since coarse-grid simulations of a whole ice sheet accounting for ice evolving anisotropy are not yet available, the choice of conditions to apply at the lateral boundary of the local flow domain is no more straightforward.

The local flow model used in this study is a multi-scale model: it provides the velocity and fabric fields correspond-

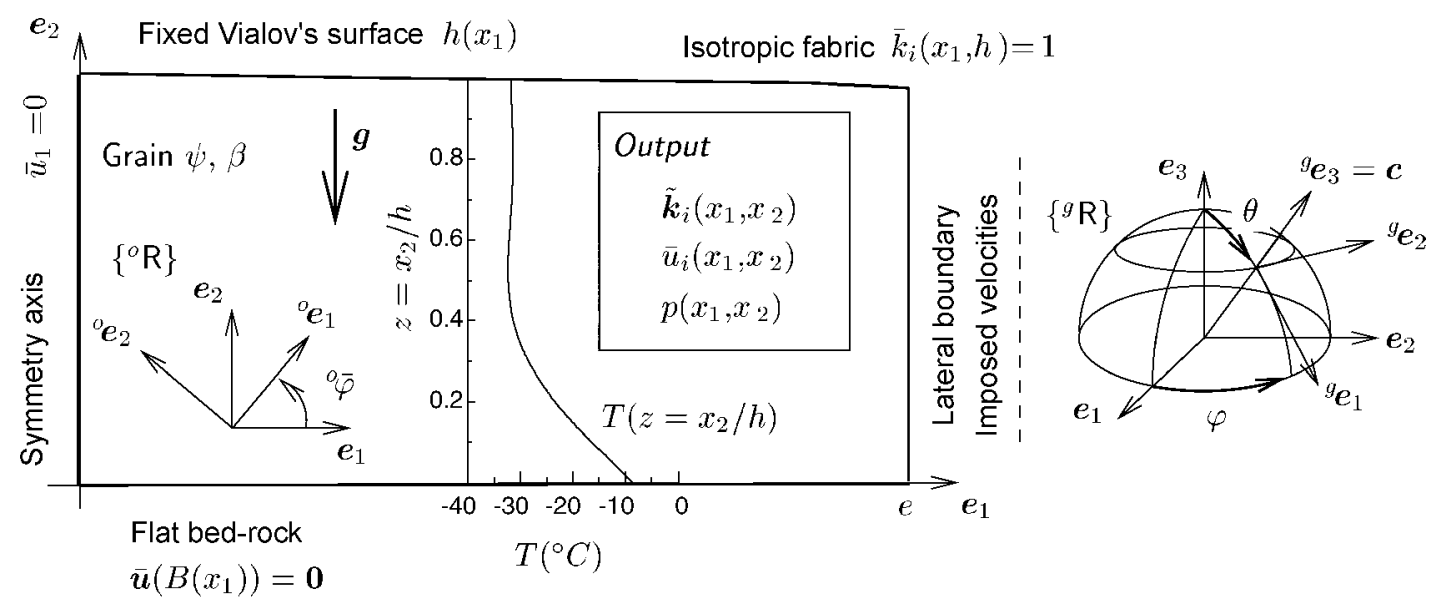

Fig. 1. Problem description and notation. For clarity, the fixed reference frame used to define the grain-reference frame is drawn rotated. 
ing to an assumed stationary state, while the behaviour of ice is obtained by a homogenization procedure which allows us to derive the polycrystal behaviour from the known behaviour of its constituent grains. The present paper focuses on the difficulties which arise when coupling this local model for the flow of orthotropic ice with a global flow model. To this end, different boundary conditions inferred from the zero-order SIA solutions for isotropic and orthotropic ice are applied at the lateral side of a two-dimensional ice sheet with simple geometry (the other lateral boundary is the dome axis), and the influence of these boundary conditions on the local flow is discussed.

\section{MODEL FOR THE ANISOTROPIC BEHAVIOUR OF ICE}

The mechanical behaviour of a polycrystal of ice is obtained by homogenization. Therefore, the polycrystal response depends on the behaviour of its grains and on their crystallographic orientations.

\section{Grain behaviour}

Following Meyssonnier and Philip (1996), each grain is assumed to behave as a linear transversely isotropic medium with rotational symmetry axis in the direction of the grain $c$ axis (the plane of isotropy is the basal plane). The local reference frame attached to a grain, with its $x_{3}$ axis along the grain $c$ axis, is denoted by $\left\{{ }^{g} \mathbf{R}\right\}$. Since each grain is transversely isotropic, its crystallographic orientation is given by the two angles $\theta$ and $\varphi$ which define the $c$-axis direction in the global reference frame $\{\boldsymbol{R}\}$ (see Fig. 1). The grain constitutive law is written in the objective form:

$$
\boldsymbol{d}=\frac{\psi}{2}\left[\beta \boldsymbol{s}+(1-\beta)\left(\boldsymbol{M}_{3} \boldsymbol{s}+\boldsymbol{s} \boldsymbol{M}_{3}-2 \operatorname{tr}\left(\boldsymbol{M}_{3} \boldsymbol{s}\right) \boldsymbol{M}\right)\right],
$$

where $\boldsymbol{M}_{3}$ is the structure tensor defined by $\boldsymbol{M}_{3}=\boldsymbol{c} \otimes \boldsymbol{c}, \boldsymbol{c}$ being the grain $c$-axis unit vector $\left({ }^{\mathrm{g}} \mathbf{c}=(0,0,1)\right.$ in $\left.\left\{{ }^{\mathrm{g}} \mathbf{R}\right\}\right)$, and $\boldsymbol{d}$ and $\boldsymbol{s}$ are the strain-rate and the deviatoric-stress tensors, respectively. Written in the grain reference frame $\left\{{ }^{g} \mathbf{R}\right\}$, the objective expression (1) takes the more usual form:

$$
\begin{aligned}
d_{i i} & =\frac{\psi}{2} \beta s_{i i}, \quad i=1,2,3, \\
d_{23} & =\frac{\psi}{2} s_{23}, \quad d_{31}=\frac{\psi}{2} s_{31}, \quad d_{12}=\frac{\psi}{2} \beta s_{12} .
\end{aligned}
$$

The parameter $\psi$ is the fluidity, inverse of viscosity, for shear parallel to the basal plane of the grain, and $\beta$ is the ratio of the shear fluidity in the basal plane to that parallel to the basal plane. $\beta$ acts as a measure of the grain anisotropy: when $\beta=0$ the grain can deform only by basal glide, as assumed in many models (Lliboutry, 1993; Van der Veen and Whillans, 1994; Mangeney and others, 1997; Gödert and Hutter, 1998), while $\beta=1$ corresponds to an isotropic grain. Since the ice single crystal deforms mainly by shear parallel to its basal plane (Duval and others, 1983), the value of $\beta$ should be significantly less than 1 . Note that the grain behaviour differs from the ice single crystal behaviour since a grain in a polycrystal interacts with its neighbours. As a consequence, the grain parameters differ from the single crystal parameters which could be derived from direct experiments. On the other hand, since the uniform-stress model does not take into account grain-to-grain interactions (it considers each grain as isolated) the grain parameters have to be fitted so that the model for a polycrystal based on the grain model reproduces experimental data.

\section{Orthotropic polycrystal behaviour}

The fabric of the ice polycrystal is described by an orientation distribution function $f(\theta, \varphi)(\mathrm{ODF})$ which gives the relative density of grains whose $c$ axes have the orientation $(\theta, \varphi)$ in the global reference frame $\{\mathbf{R}\}$. The relative number of grains with orientation $(\theta, \varphi)$ is $f(\theta, \varphi) \sin \theta$. Since recrystallization is not accounted for, fabric evolution is solely due to grain lattice rotation, i.e. the net flux of grains entering or leaving the interval $\mathrm{d} \theta, \mathrm{d} \varphi$ at point $(\theta, \varphi)$ equals the increase in the number of grains in this interval during time increment $\mathrm{d} t$, that is

$$
\frac{\partial f \sin \theta}{\partial t}+\frac{\partial \dot{\theta} f \sin \theta}{\partial \theta}+\frac{\partial \dot{\varphi} f \sin \theta}{\partial \varphi}=0 \text {. }
$$

The grain rotation rates $\dot{\theta}$ and $\dot{\varphi}$ in Equation (3) are determined from the decomposition of the spin of each grain into a component due to its visco-plastic deformation (measured in the grain reference frame $\left\{{ }^{\mathrm{g}} \mathbf{R}\right\}$ ) plus a component corresponding to the rotation of the basal planes (Meyssonnier and Philip, 1996). The homogenization procedure to derive the polycrystal behaviour is based on the assumption of a uniform state of stress in the polycrystal, i.e. the stress in any grain is the same as the stress in the aggregate considered as a homogeneous medium (this model is often referred to as "static model"). Using the overbar symbol to denote quantities defined at the polycrystal (macroscopic) scale, this condition is expressed simply as:

$$
\boldsymbol{s}=\overline{\boldsymbol{s}}
$$

According to Gagliardini and Meyssonnier (1999a) and assuming that the spin of each grain, expressed as $\boldsymbol{w}$ in the global reference frame $\{\mathbf{R}\}$, equals the macroscopic spin of the polycrystal $\overline{\boldsymbol{w}}$ (Taylor-type assumption) the grain rotation rates $\dot{\theta}$ and $\dot{\varphi}$ corresponding to plane flow can be expressed in terms of the macroscopic deviatoric-stress components $\bar{s}_{11}, \bar{s}_{22}, \bar{s}_{12}$ and of the macroscopic spin component $\bar{w}_{12}$ as

$$
\begin{aligned}
& \dot{\theta}=-\frac{\psi}{8} \sin 2 \theta\left[3\left(\bar{s}_{11}+\bar{s}_{22}\right)+\left(\bar{s}_{11}-\bar{s}_{22}\right) \cos 2 \varphi+2 \bar{s}_{12} \sin 2 \varphi\right] \\
& \dot{\varphi}=\frac{\psi}{4}\left[\left(\bar{s}_{11}-\bar{s}_{22}\right) \sin 2 \varphi-2 \bar{s}_{12} \cos 2 \varphi\right]-\bar{w}_{12}
\end{aligned}
$$

Equations (3) and (5) describe completely the evolution of the ice fabric in the particular case of plane flow.

In what follows, we assume that the fabric can be described by a parameterized ODF which was derived from analytical calculations by Gagliardini and Meyssonnier (1999a) under the assumptions that the principal stress directions are fixed and that $\bar{w}_{12}=0$. This parameterized ODF is given by

$$
\begin{aligned}
f\left(\theta, \varphi, \bar{k}_{1}, \bar{k}_{2},{ }^{\mathrm{o}} \bar{\varphi}\right)= & \left\{\operatorname { s i n } ^ { 2 } \theta \left[k_{1}^{2} \cos ^{2}\left(\varphi-{ }^{\circ} \bar{\varphi}\right)\right.\right. \\
& \left.\left.+\bar{k}_{2}^{2} \sin ^{2}\left(\varphi-{ }^{o} \bar{\varphi}\right)\right]+\bar{k}_{3}^{2} \cos ^{2} \theta\right\}^{-3 / 2} .
\end{aligned}
$$

Since the equation for the conservation of the total number of grains,

$$
\frac{1}{2 \pi} \int_{0}^{2 \pi} \int_{0}^{\pi / 2} f(\theta, \varphi) \sin \theta \mathrm{d} \theta \mathrm{d} \varphi=1,
$$


implies

$$
\bar{k}_{1} \bar{k}_{2} \bar{k}_{3}=1 \text {, }
$$

the ODF (Equation (6)) depends only on three independent parameters $\bar{k}_{1}, \bar{k}_{2}$ and ${ }^{\circ} \bar{\varphi}$. Relation (6) describes an orthotropic fabric with planes of symmetry $\left({ }^{\circ} x_{1},{ }^{\circ} x_{2}\right),\left({ }^{\circ} x_{2},{ }^{\circ} x_{3}\right)$ and $\left({ }^{\circ} x_{3},{ }^{\circ} x_{1}\right)$. In the following, the plane $\left({ }^{\mathrm{o}} x_{1},{ }^{\circ} x_{2}\right)$ coincides with the plane $\left(x_{1}, x_{2}\right)$ of the ice-sheet flow (see Fig. 1). Each parameter $\bar{k}_{i}$ gives the strength of concentration of $c$ axes in the direction ${ }^{\circ} \boldsymbol{e}_{i}$ of the material symmetry reference frame $\left\{{ }^{\circ} \mathbf{R}\right\}$ attached to the polycrystal (defined by the three orthogonal planes of orthotropy). A small value of $\bar{k}_{i}$ corresponds to $c$ axes gathered along direction ${ }^{\circ} \boldsymbol{e}_{i}$. The angle ${ }^{\circ} \bar{\varphi}$ defines the rotation around the $x_{3}$ axis (in the plane $\left.\left(x_{1}, x_{2}\right)\right)$ of $\left\{{ }^{\mathrm{o}} \mathbf{R}\right\}$ with respect to the global reference frame $\{\mathbf{R}\}$ (see Fig. 1).

Taking Equation (4) into account, the constitutive law for the orthotropic polycrystal with a fabric given by Equation (6) is obtained by expressing the macroscopic strain rate as the weighted average of the grain strain rates given by Equation (1), that is

$$
\overline{\boldsymbol{d}}=\langle\boldsymbol{d}\rangle
$$

where

$$
\langle\boldsymbol{d}\rangle=\frac{1}{2 \pi} \int_{0}^{2 \pi} \int_{0}^{\pi / 2} \boldsymbol{d}(\theta, \varphi) f(\theta, \varphi) \sin \theta \mathrm{d} \theta \mathrm{d} \varphi .
$$

For flow numerical solving, it is convenient to use the inverted form of the constitutive law derived from Equation (9) which is found as

$$
\overline{\boldsymbol{s}}=\sum_{i=1}^{3}\left[\bar{\eta}_{i} \operatorname{tr}\left({ }^{\mathrm{o}} \overline{\boldsymbol{M}}_{i} \overline{\boldsymbol{d}}\right)^{\mathrm{o}} \overline{\boldsymbol{M}}_{i}^{D}+\bar{\eta}_{i+3}\left(\overline{\boldsymbol{d}}^{\mathrm{o}} \overline{\boldsymbol{M}}_{i}+{ }^{\mathrm{o}} \overline{\boldsymbol{M}}_{i} \overline{\boldsymbol{d}}\right)^{D}\right]
$$

where $\bar{\eta}_{i}$ are macroscopic viscosities which are expressed in terms of the grain rheological parameters $\psi$ and $\beta$ and of fabric parameters $\bar{k}_{i}$ and ${ }^{\circ} \bar{\varphi}$ (see Gagliardini and Meyssonnier, 1999b, for these relations), and ${ }^{\circ} \overline{\boldsymbol{M}}_{i}={ }^{\mathrm{o}} \boldsymbol{e}_{i} \otimes{ }^{\circ} \boldsymbol{e}_{i}$ are three structure tensors defined by the unit vectors ${ }^{\circ} \boldsymbol{e}_{i}$ of $\left\{{ }^{\circ} \mathbf{R}\right\}$. For isotropic ice, i.e. when $f(\theta, \varphi) \equiv 1$ and $\bar{k}_{1}=\bar{k}_{2}=\bar{k}_{3}=1, \bar{\eta}_{i}=$ 0 and $\bar{\eta}_{i+3}=1 / \bar{B}_{1}$ for $i=1,2,3$, so that Equation (11) reduces to a linearly viscous law $\overline{\boldsymbol{d}}=\bar{B}_{1} \overline{\boldsymbol{s}} / 2$ corresponding to the linear version of Glen's law. The fluidity parameter $\bar{B}_{1}$ is related to the grain rheological parameters by Gagliardini and Meyssonnier (1999a):

$$
\bar{B}_{1}=\frac{\psi}{5}(3 \beta+2) \text {. }
$$

$\bar{B}_{1}$, then $\psi$ according to Equation (12), are temperaturedependent and follow the Arrhenius law

$$
\bar{B}_{1}(T)=\bar{B}_{1}\left(T_{0}\right) \mathrm{e}^{Q\left(1 / T_{0}-1 / T\right) / R},
$$

where $Q$ is the activation energy, $R$ is the gas constant and $T, T_{0}$ are temperatures in Kelvin.

\section{FLOW AND FABRIC EVOLUTION PROBLEMS}

At a given material point $\boldsymbol{x}$ in the ice sheet the ice fabric is given by Equation (6) in which parameters $\bar{k}_{i}$ and ${ }^{\circ} \varphi$ are $\boldsymbol{x}$ dependent. In the following, taking Equation (8) into account, the fabric is described by using the threecomponent fabric vector $\tilde{\overline{\boldsymbol{k}}}(\boldsymbol{x})=\left(\bar{k}_{1}(\boldsymbol{x}), \bar{k}_{2}(\boldsymbol{x}),{ }^{\circ} \bar{\varphi}(\boldsymbol{x})\right)$.

Then, the problems to be solved are (i) for a given fabric field $\tilde{\boldsymbol{k}}(\boldsymbol{x})$, the gravity-driven plane flow for fixed surface and bedrock topographies, and (ii) for a given velocity field $\overline{\mathbf{u}}(\boldsymbol{x})$, the fabric field assuming an isotropic fabric at the icesheet surface and stationary flow. The velocity and fabric fields corresponding to stationary flow are calculated by iteratively solving the velocity problem and the fabric problem, until convergence is achieved.

In this study we adopt a simplified ice-sheet geometry, with a flat bedrock and a fixed surface elevation given by Vialov's profile (Vialov, 1958):

$$
h\left(x_{1}\right)^{4}=h_{0}^{4}\left[1-\left(\frac{x_{1}}{L}\right)^{2}\right]
$$

where $h_{0}$ is the ice depth at the dome and $L$ is the ice-sheet length. Note that the local flow model is applied on a small part of the ice sheet delimited by the dome $x_{1}=0$ and a fictitious vertical boundary at $x_{1}=e$.

\section{Flow equations}

For the flow problem, the micro-macro polycrystal law (Equation (11)) is incorporated into a finite-element code in order to solve the quasi-static stress-equilibrium equations corresponding to plane strain flow

$$
\frac{\partial \bar{s}_{11}}{\partial x_{1}}+\frac{\partial \bar{s}_{12}}{\partial x_{2}}+\frac{\partial \bar{p}}{\partial x_{1}}=0, \quad \frac{\partial \bar{s}_{12}}{\partial x_{1}}+\frac{\partial \bar{s}_{22}}{\partial x_{2}}+\frac{\partial \bar{p}}{\partial x_{2}}=\rho g,
$$

where $\bar{p}=\operatorname{tr}() / 3$ is the isotropic pressure and $\rho g$ is the gravity force (per unit volume), and the incompressibility equation

$$
\frac{\partial \bar{u}_{1}}{\partial x_{1}}+\frac{\partial \bar{u}_{2}}{\partial x_{2}}=0
$$

where $\bar{u}_{i}$ is the velocity component in direction $\boldsymbol{e}_{i}$ of $\{\mathbf{R}\}$.

Note that since the surface elevation is fixed, the accumulation rate $\bar{b}$ must be considered as a variable depending on the solution of the flow problem which derives from mass conservation as

$$
\bar{b}\left(x_{1}\right)=\bar{u}_{1}\left(x_{1}, h\right) \frac{\partial h}{\partial x_{1}}-\bar{u}_{2}\left(x_{1}, h\right) .
$$

The boundary conditions of the flow problem are:

No sliding at the ice-bedrock interface $x_{2}=0$, hence

$$
\bar{u}_{i}\left(x_{1}, 0\right)=0 \quad i=1,2 .
$$

The vertical axis of symmetry of the two-dimensional ice sheet is assumed to be at the dome $x_{1}=0$ (see Fig. 1) which implies

$$
\bar{u}_{1}\left(0, x_{2}\right)=0 \text { and } \quad \bar{s}_{12}\left(0, x_{2}\right)=0 .
$$

A boundary condition has to be imposed at the lateral boundary $x_{1}=e$.

Different kinds of Dirichlet boundary conditions are studied below.

From a numerical point of view, the isotropic pressure is used as a Lagrange multiplier in order to solve the incompressibility equation (16). The mesh is made of six-node triangular elements, with a quadratic interpolation of the velocities and a linear interpolation of the isotropic pressure. For the flow problem, the fabric parameters $\bar{k}_{1}, \bar{k}_{2}$ and ${ }^{\circ} \bar{\varphi}$ are given at each node of the mesh and interpolated quadratically.

\section{Fabric evolution}

The problem is to find the fabric field $\tilde{\overline{\boldsymbol{k}}}(\boldsymbol{x})$ for a given velocity field $\overline{\boldsymbol{u}}(\boldsymbol{x})$. The conservation equation for the grain 
orientations written in the form of Equation (3) supposes that the polycrystal is followed along its trajectory (Lagrangian point of view). From the Eulerian point of view adopted here to solve the stationary problem, the term $\partial(f \sin \theta) / \partial t$ is zero and must be replaced by a convective term $\left[\partial(f \sin \theta) / \partial x_{i}\right] \bar{u}_{i}$ (at point $\boldsymbol{x}$ the observed polycrystal comes from upstream). Then the ODF evolution equation (3) becomes

$$
\sum_{j=1}^{3} \frac{\partial f \sin \theta}{\partial \tilde{\bar{k}}_{j}} \frac{\partial \tilde{\bar{k}}_{j}}{\partial x_{i}} \bar{u}_{i}+\frac{\partial \dot{\theta} f \sin \theta}{\partial \theta}+\frac{\partial \dot{\varphi} f \sin \theta}{\partial \varphi}=0 .
$$

Using Expressions (5) for $\dot{\theta}$ and $\dot{\varphi}$ and (6) for $f$, Equation (20) simplifies as

$$
\frac{\partial f}{\partial \tilde{\bar{k}}_{i}} Q_{i}+\frac{\partial \dot{\theta} f}{\partial \theta}+\dot{\Theta} f \cos \theta+\frac{\partial \dot{\varphi} f}{\partial \varphi}=0,
$$

where $Q_{i}=Q\left(\tilde{\bar{k}}_{i}\right)=\left(\partial \tilde{\bar{k}}_{i} / \partial x_{j}\right) \bar{u}_{j}$ for $i=1,2,3$, and where $\dot{\Theta}$ is such that $\dot{\theta}=\dot{\Theta} \sin \theta$. That is, from Equation (5) 1 ,

$\dot{\Theta}=-\frac{\psi}{4} \cos \theta$

$$
\cdot\left[3\left(\bar{s}_{11}+\bar{s}_{22}\right)+\left(\bar{s}_{11}-\bar{s}_{22}\right) \cos 2 \varphi+2 \bar{s}_{12} \sin 2 \varphi\right] .
$$

Equation (21) is valid at each point $\boldsymbol{x}$ whatever the grain orientation $(\theta, \varphi)$ considered. Three independent evolution equations for the ODF parameters are obtained from Equation (21) by following the method proposed by Gödert and Hutter (2000). First, since $\partial f / \partial \bar{k}_{2}$ and $\partial f / \partial^{\circ} \bar{\varphi}$ are zero for $\theta=\pi / 2$ and $\varphi={ }^{\circ} \bar{\varphi}$, replacing $(\theta, \varphi)$ by $\left(\pi / 2,{ }^{\circ} \bar{\varphi}\right)$ in Equation (21) provides $Q_{1}=Q\left(\bar{k}_{1}\right)$. Second, $\partial f / \partial^{\circ} \bar{\varphi}$ being zero for $\theta=0$, making $\theta=0$ in Equation (21) provides $Q_{1}+Q_{2}$, then using the first relation $Q_{2}=Q\left(\bar{k}_{2}\right)$. Finally $Q_{3}=Q\left({ }^{\circ} \bar{\varphi}\right)$ is simply derived from Equation (21) by replacing $Q_{1}$ and $Q_{2}$ in Equation (21) by their expressions so obtained. The resulting three evolution equations for the ODF parameters are:

$$
\begin{aligned}
& \frac{\partial \bar{k}_{1}}{\partial x_{i}} \bar{u}_{i}-\bar{k}_{1} \frac{\psi}{4} \\
& \cdot\left[\bar{s}_{11}+\bar{s}_{22}+\left(\bar{s}_{11}-\bar{s}_{22}\right) \cos 2^{\mathrm{o}} \bar{\varphi}+2 \bar{s}_{12} \sin 2^{\mathrm{o}} \bar{\varphi}\right]=0, \\
& \frac{\partial \bar{k}_{2}}{\partial x_{i}} \bar{u}_{i}-\bar{k}_{2} \frac{\psi}{4} \\
& \cdot\left[\bar{s}_{11}+\bar{s}_{22}-\left(\bar{s}_{11}-\bar{s}_{22}\right) \cos 2^{\mathrm{o}} \bar{\varphi}-2 \bar{s}_{12} \sin 2^{\mathrm{o}} \bar{\varphi}\right]=0, \\
& \frac{\partial^{\mathrm{o}} \bar{\varphi}}{\partial x_{i}} \bar{u}_{i}-\frac{\psi}{4} \frac{1+\left(\bar{k}_{2} / \bar{k}_{1}\right)^{2}}{1-\left(\bar{k}_{2} / \bar{k}_{1}\right)^{2}} \\
& \cdot\left[2 \bar{s}_{12} \cos 2^{\mathrm{o}} \bar{\varphi}-\left(\bar{s}_{11}-\bar{s}_{22}\right) \sin 2^{\mathrm{o}} \bar{\varphi}\right]+\bar{w}_{12}=0
\end{aligned}
$$

In a previous model (Gagliardini and Meyssonnier, 1999a, 2000) fabric evolution was calculated differently. On the one hand, the $\bar{k}_{i}$ parameters were obtained analytically under the assumptions that the directions of the principal stresses do not change significantly along a streamline during the time-step $\mathrm{d} t$. On the other hand, the increment of rotation of the material symmetry reference $\mathrm{d}^{\mathrm{o}} \bar{\varphi}$ was taken as $\langle\dot{\varphi}\rangle \mathrm{d} t$ which required an integration over $\theta$ and $\varphi$ at each time-step. Compared to this previous model, the fabric evolution problem is now notably simplified and also solved in a more rigorous way.

Numerically, Equation (23) is solved by using a secondorder Runge-Kutta method along the streamlines derived from the solution of the flow problem. Since the ice deposited on the ice-sheet surface is assumed to be isotropic, the boundary conditions on fabric parameters $\bar{k}_{i}$ are

$$
\bar{k}_{i}\left(x_{1}, h\right)=1 \quad i=1,2,3,
$$

while ${ }^{\circ} \bar{\varphi}\left(x_{1}, h\right)$ is determined by the principal stress directions at the surface.

\section{LATERAL BOUNDARY GONDITIONS}

Different Dirichlet-type boundary conditions (i.e. given values of $\left.\bar{u}_{i}\left(e, x_{2}\right)\right)$ were applied at the lateral boundary $x_{1}=e$ of the model. These conditions are inferred from analytical developments of the zero-order SIA for isotropic and orthotropic ice.

\section{SIA formulation for orthotropic ice}

In the framework of the SIA (see, e.g., Hutter, 1983), since the length of the ice sheet $L$ is much larger than its depth $h_{0}$ at the dome, the small parameter $\epsilon=h_{0} / L$ is used to expand the flow Equations (11), (15) and (16) in power series of $\epsilon$. Using capital letters for stretched variables, the actual coordinates $x_{1}, x_{2}$ and surface elevation $h$ transform respectively into $X_{1}, X_{2}$ and $H$, such that:

$$
X_{1}=\epsilon x_{1}, \quad X_{2}=x_{2}, \quad H=h .
$$

According to Mangeney and Califano (1998) the stretched velocities, strain-rate and deviatoric-stress components are defined as:

$$
\begin{aligned}
& \bar{U}_{1}=\epsilon \bar{u}_{1}, \quad \bar{U}_{2}=\bar{u}_{2} \\
& \bar{D}_{11}=\bar{d}_{11}, \quad \bar{D}_{22}=\bar{d}_{22}, \quad 2 \bar{D}_{12}=\frac{1}{\epsilon} \frac{\partial \bar{U}_{1}}{\partial X_{2}}+\epsilon \frac{\partial \bar{U}_{2}}{\partial X_{1}} \\
& \bar{P}=\bar{p}, \quad \bar{S}_{i j}=\bar{s}_{i j} / \epsilon .
\end{aligned}
$$

The SIA equations are derived by using the linear orthotropic law (Equation (11)). Following Mangeney and Califano (1998), a coherent stretching of the viscosities $\bar{\eta}_{i}$ in Equation (11) is chosen as

$$
\overline{\boldsymbol{M}}_{i}=\frac{\bar{\eta}_{i}}{\epsilon^{2}}
$$

Following Philip and Meyssonnier (1999), taking into account Equations (25-27), the equations of the flow problem (15) and (16) at order $\mathcal{O}\left(\epsilon^{0}\right)$ for orthotropic ice behaviour are

$$
\begin{array}{ll}
\bar{S}_{11}=\overline{\mathcal{M}}_{1112} \frac{\partial \bar{U}_{2}}{\partial X_{1}}, & \bar{S}_{22}=\overline{\mathcal{M}}_{2212} \frac{\partial \bar{U}_{2}}{\partial X_{1}}, \\
\bar{S}_{33}=\overline{\mathcal{M}}_{3312} \frac{\partial \bar{U}_{2}}{\partial X_{1}}, & \bar{S}_{12}=\overline{\mathcal{M}}_{1212} \frac{\partial \bar{U}_{2}}{\partial X_{1}}, \\
\frac{\partial \bar{P}}{\partial X_{1}}+\frac{\partial \bar{S}_{12}}{\partial X_{2}}=0, \quad \frac{\partial \bar{P}}{\partial X_{2}}=\rho g, \\
\frac{\partial \bar{U}_{1}}{\partial X_{1}}+\frac{\partial \bar{U}_{2}}{\partial X_{2}}=0,
\end{array}
$$

where $\overline{\mathcal{M}}_{i j 12}$ are the stretched polycrystal viscosities expressed in the global reference frame $\{\mathbf{R}\}$. For the orthotro- 
pic behaviour (Equation (11)) considered, they are found to be of the form

$$
\begin{aligned}
& \overline{\mathcal{M}}_{1112}=\sin 2^{\mathrm{o}} \bar{\varphi} \\
& \cdot \frac{3\left(\overline{\mathcal{M}}_{1}+\overline{\mathcal{M}}_{2}\right) \cos ^{2}{ }^{\mathrm{o}} \bar{\varphi}-\left(\overline{\mathcal{M}}_{1}+2 \overline{\mathcal{M}}_{2}-\overline{\mathcal{M}}_{4}+\overline{\mathcal{M}}_{5}\right)}{6} \\
& \overline{\mathcal{M}}_{2212}=\sin 2^{\mathrm{o}} \bar{\varphi} \\
& \cdot \frac{-3\left(\overline{\mathcal{M}}_{1}+\overline{\mathcal{M}}_{2}\right) \cos ^{2}{ }^{\mathrm{o}} \bar{\varphi}+\left(2 \overline{\mathcal{M}}_{1}+\overline{\mathcal{M}}_{2}+\overline{\mathcal{M}}_{4}-\overline{\mathcal{M}}_{5}\right)}{6} \\
& \overline{\mathcal{M}}_{3312}=\sin 2^{\mathrm{o}} \bar{\varphi} \frac{-\overline{\mathcal{M}}_{1}+\overline{\mathcal{M}}_{2}-2 \overline{\mathcal{M}}_{4}+2 \overline{\mathcal{M}}_{5}}{6} \\
& \overline{\mathcal{M}}_{1212}=\frac{\sin ^{2} 2^{\mathrm{o}} \bar{\varphi}\left(\overline{\mathcal{M}}_{1}+\overline{\mathcal{M}}_{2}\right)+4 \overline{\mathcal{M}}_{4}+4 \overline{\mathcal{M}}_{5}}{4}
\end{aligned}
$$

where $\overline{\mathcal{M}}_{i}$ is given by Equation (27).

Using Equations (28), (29) and (27), the form of the zeroorder solution is found as

$$
\begin{gathered}
\bar{P}=\rho g\left(X_{2}-H\right), \\
\bar{S}_{12}=\rho g H^{\prime}\left(X_{2}-H\right), \\
\frac{\partial \bar{U}_{1}}{\partial X_{2}}=\frac{\rho g}{\overline{\mathcal{M}}_{1212}} H^{\prime}\left(X_{2}-H\right), \\
\frac{\partial^{2} \bar{U}_{2}}{\partial X_{2}^{2}}=-\frac{\rho g}{\overline{\mathcal{M}}_{1212}}\left[H^{\prime \prime}\left(X_{2}-H\right)-\left(H^{\prime}\right)^{2}\right],
\end{gathered}
$$

where $H^{\prime}$ and $H^{\prime \prime}$ are the first and second derivative of $H$ with respect to $X_{1}$, respectively.

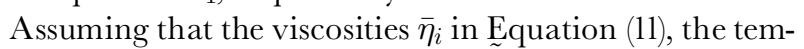
perature $T$ and the fabric parameters $\tilde{\overline{\boldsymbol{k}}}$ are functions only of the reduced depth $z=x_{2} / h\left(x_{1}\right)$, Equations (30) $)_{3}$ and (30) 4 are solved at the lateral boundary of the local model $x_{1}=e$, using a second-order Runge-Kutta method, with boundary conditions $\bar{u}_{i}(e, 0)=0$ and $\partial \overline{u_{2}} / \partial x_{2}(e, 0)=0$.

\section{The different boundary conditions}

Three kinds of lateral boundary conditions at $x_{1}=e$ are considered:

The "isotropic" boundary condition is derived from the SIA solution of Equation (30) for $\bar{u}_{i}\left(e, x_{2}\right)$, assuming isotropic behaviour of ice. Then the viscosities in Equation (11) are $\bar{\eta}_{i}=0$ and $\bar{\eta}_{i+3}=1 / \bar{B}_{1}$ for $i=1,2,3$, which implies that $\overline{\mathcal{M}}_{i i 12}=0$ for $i=1,2,3$ and $\overline{\mathcal{M}}_{1212}=1 /\left(\epsilon^{2} \bar{B}_{1}\right)$ in Equation (30). Since we assume a non-uniform field of temperature of the form $T=T(z)$, there is no analytical solution for these velocities.

The "orthotropic" boundary condition is derived from the SIA solution of Equation (30), assuming orthotropic behaviour of ice. The vertical fabric profile used to derive the ice behaviour (Equation (11)) needed for the SIA calculations is the one calculated at the lateral boundary of the local flow model at $x_{1}=e$, i.e. $\tilde{\overline{\boldsymbol{k}}}(z)$ used in the SIA calculations equals $\overline{\boldsymbol{k}}\left(e, x_{2}\right)$ resulting from the local flow model. Note that, unlike the "isotropic" boundary condition case, the finite-element local flow problem and the SIA calculations are coupled: the vertical fabric profile $\tilde{\overline{\boldsymbol{k}}}(z)$ used in the SIA

\begin{tabular}{|c|c|}
\hline$\beta=0.25$ & Grain anisotropy parameter in Equation (1) \\
\hline$Q=78000 \mathrm{~J} \mathrm{~mol}^{-1}$ & Activation energy \\
\hline$R=8.314 \mathrm{~J} \mathrm{~mol}^{-1} \mathrm{~K}^{-1}$ & Gas constant \\
\hline
\end{tabular}
calculation changes at each step of the coupled problem, while the boundary condition at $x_{1}=e$ must be recalculated before each local flow computation.

The "enhanced isotropic" boundary condition is the same as the "isotropic" one, but the imposed velocities are scaled by an enhancement factor $E$, constant with depth, calculated to match the horizontal velocity of the "orthotropic" bound-
Table 1. Value of the grain parameters used in finite-element computations

ary condition at the top of the lateral boundary. The SIA solution is obtained by using the enhanced fluidity $E / \overline{\mathcal{M}}_{1212}$ instead of $1 / \overline{\mathcal{M}}_{1212}$ in Equation (30).

\section{LOGAL-FLOW MODEL RESULTS}

\section{Input data for numerical simulations}

In order to assess the influence of the boundary condition applied at the lateral boundary, the study is restricted to results obtained on a simplified two-dimensional ice sheet. All the calculations were performed under the following common assumptions (see Fig. 1):

The fixed surface elevation is given by Vialov's profile (Equation (14)) with $\epsilon=0.005\left(L=200 h_{0}\right)$, and the bedrock is assumed to be perfectly flat.

The temperature field is deduced from the Greenland Icecore Project (GRIP) borehole measurements (Gundestrup and others, 1993), assuming that the temperature is a function of the reduced elevation $z$ only (i.e. independent of $x_{1}$ ). The temperature increases by $23.3^{\circ} \mathrm{C}$ from the surface $\left(-31.7^{\circ} \mathrm{C}\right)$ to the bottom $\left(-8.4^{\circ} \mathrm{C}\right)$.

The studied domain is restricted to $0 \leq x \leq e$ with $e 20$ times the depth at the dome, i.e. $e=20 h_{0}=0.1 L$.

Adimensional velocities are obtained by using $\bar{B}_{1} \rho g h_{0}^{2}$ as velocity unit.

The numerical values assigned to the grain behaviour parameters (except $\bar{B}_{1}$ ) are given in Table 1 . They were obtained by comparison of the polycrystal model results with field data from the GRIP ice core (Gagliardini and Meyssonnier, 1999a).

The finite-element mesh is composed of 20 horizontal layers and 60 vertical columns of quadrilateral domains, each subdivided into four triangular six-node elements. This corresponds to 4800 triangles and 9761 nodes. The mesh is refined near the ice divide and near the bedrock.

\section{Results}

The results obtained for the convergence of the local coupled flow problem are the velocity and the fabric fields corresponding to the stationary flow of an ice sheet with fixed geometry.

From a numerical point of view, convergence of the coupled problem (i.e. computation of the velocity field and fabric field corresponding to stationary state) for both the "isotropic" and "enhanced isotropic" boundary conditions was obtained after more than 30 iterations, whereas the convergence was obtained after only 10 iterations for the "orthotropic" boundary condition.

As expected from Mangeney and others (1996), the imposed velocities at the lateral boundary with the "isotropic" boundary 

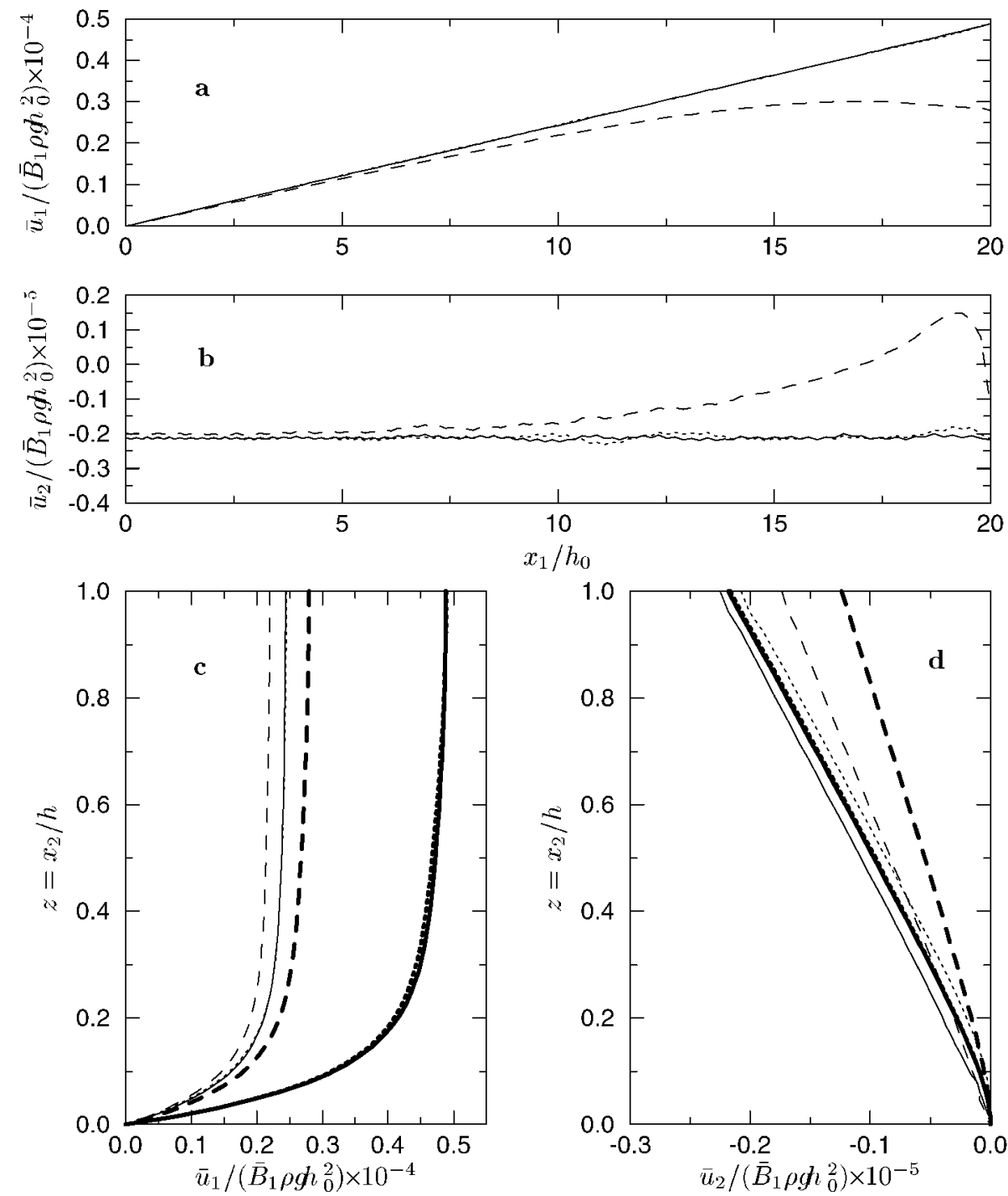

Fig. 2. (a) Horizontal and (b) vertical velocities on the ice-sheet surface; (c) horizontal and (d) vertical velocity profiles at $x_{1}=10 h_{0}$ (thin curves) and at $x_{1}=20 h_{0}=e$ (thick curves) for the "isotropic" (dashed line), "orthotropic" ( solid line) and "enhanced isotropic" (dotted line) boundary conditions.

condition are smaller by a factor of 1.75 than that obtained with the "orthotropic" boundary condition (see Fig. 2c and d). Consequently the "isotropic" boundary condition slows down the flow. The influence of such a constraint is visible on the surface velocities: owing to ice incompressibility, the vertical velocity becomes positive (upward direction) just upstream of the lateral boundary $x_{1}=e$ (for a given accumulation rate this would correspond to a bump of the free surface).

On the other hand, the "orthotropic" boundary condition seems to have only a very weak influence on the velocities. As shown by Mangeney and Califano (1998) for transversely isotropic ice, the first- and second-order terms of the SIA solution are negligible compared to the zeroorder terms. This could explain why the local velocities are not disturbed by the lateral boundary condition. The vertical velocities are slightly oscillating around a mean value (see Fig. 2b) which is attributed to the imposed velocities at the lateral boundary.

The enhancement factor for the "enhanced isotropic" boundary condition is taken as $E=1.75$, so that the horizontal velocity $\bar{u}_{1}(e, h)$ at the ice-sheet surface is equal to that corresponding to the "orthotropic" boundary condition. Owing to the imposed temperature profile $T(z)$ (temperature increase of $23.3^{\circ} \mathrm{C}$ from surface to bottom corresponding to an increase of the ice fluidity by a factor about 30), most of the deformation is located near the bedrock. As a consequence, although the two SIA solutions are theoretically different (the orthotropic SIA solution accounts for a variation of $\overline{\mathcal{M}}_{1212}$ with depth, whereas the enhanced isotropic solution assumes a constant viscosity), both the horizontal and vertical imposed velocity profiles of the "enhanced isotropic" and of the "orthotropic" boundary conditions are very close to each other. As a consequence, the velocity field obtained with the "enhanced isotropic" boundary condition is very similar to that obtained in the "orthotropic" case (see Fig. 2). For a smaller increase of the temperature with depth, the difference would be more significant.

The influence of the boundary conditions on the fabricfield results was assessed by comparing the evolutions with depth of the fabric-strength parameter $R_{0}$ and of the orientation ${ }^{\circ} \bar{\varphi}$ of the material symmetry reference frame (see Fig. 3). $R_{0}$ is a statistical parameter which indicates the strength of the fabric and is defined as:

$$
R_{0}=2\|\langle\boldsymbol{c}\rangle\|,
$$

where $\boldsymbol{c}$ is the $c$-axis unit vector and \|\| denotes the norm of a vector. An isotropic fabric corresponds to $R_{0}=0$, and $R_{0}$ takes the maximum value 1 when all the grain $c$ axes have the same orientation. As for the velocities, the fabrics obtained 

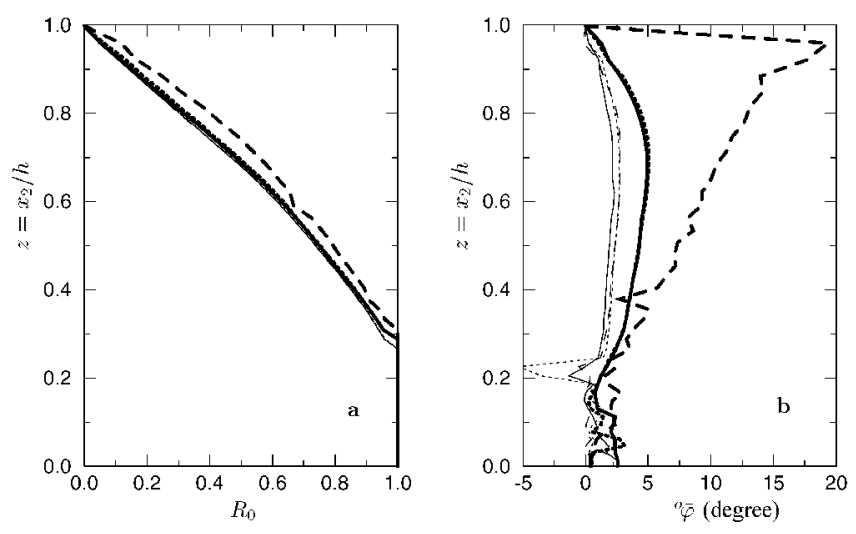

\begin{abstract}
Fig. 3. (a) Fabric-strength parameter $R_{0}$ and (b) orientation of the orthotropy reference frame as a function of reduced depth $z$ at $x_{1}=10 h_{0}$ (thincurves) and $x_{1}=20 h_{0}=e$ (thick curves) for the "isotropic" (dashed line), "orthotropic" (solid line) and "enhanced isotropic" (dotted line) boundary conditions.
\end{abstract}

with the "enhanced isotropic" and "orthotropic" boundary conditions are very similar. As shown on Figure 3, the differences on the fabric fields for the "isotropic" and "orthotropic" boundary conditions are smaller than those observed on the velocities. The larger difference is observed at the lateral boundary at $x_{1}=e=20 h_{0}$, and ${ }^{\circ} \bar{\varphi}$ is more perturbed than the strength of the fabric. A possible explanation is that since the evolution of the fabric parameters is obtained by integration of Equations (23) along the flow streamlines, it is naturally sensitive to perturbations of the flow to some extent. However, surface perturbations have much more influence on ${ }^{\circ} \bar{\varphi}$ than on parameters $\bar{k}_{i}$ because they directly influence the initial (surface) condition on ${ }^{\circ} \bar{\varphi}$ (which is determined by the principal stress directions at the surface), while the initial conditions on the fabric-streng th parameters (i.e. $\bar{k}_{i}=1$ ) are independent of the velocity.

\section{GONCLUSION}

A multi-scale model for the two-dimensional flow of polar ice exhibiting a strain-induced evolving anisotropy has been reviewed. The linear orthotropic behaviour of a polycrystal of ice was obtained analytically by homogenization assuming a uniform state of stress in the polycrystal whose crystallographic texture is described by an ODF of its $c$ axes. To simulate the stationary flow of an idealized two-dimensional ice sheet, a parameterized form of the ODF which depends on three parameters was used. However, the existing model is still too complex to be used to simulate the flow of a real ice sheet at the global scale, and potential applications are currently restricted to limited spatial domains around existing drilling sites. Then the conditions to apply on the lateral (fictitious) boundaries of the studied domain must be assessed.

In this paper, three types of lateral boundary conditions have been applied on the lateral boundary of a section of a Vialov's ice sheet resting on a flat bedrock. These Dirichlettype boundary conditions were derived from SIA solutions at order $\mathcal{O}\left(\epsilon^{0}\right)$ for isotropic and orthotropic ice. Comparison of the results shows that the lateral "orthotropic" boundary condition derived from the SIA for anisotropic ice leads to smooth velocities, whereas the "isotropic" boundary condition generates strong perturbations of the flow. The present study shows that, because Dirichlet-type conditions are too constraining, the improvement expected from a local model accounting for the physical mechanisms involved in the description of ice behaviour could be wiped out by coupling the local model with a global model which considers ice as an isotropic medium. Using an enhanced isotropic fluidity in the SIA with an appropriate enhancement factor $E$ derived from the "orthotropic" SIA solution leads to a boundary condition very close to the "orthotropic". However, in a general situation, the difficulty would be to guess the appropriate value for $E$ which is a priori unknown.

\section{AGKNOWLEDGEMENTS}

We thank two anonymous referees for their very relevant comments and suggestions for improving the first version of this paper.

\section{REFERENCES}

Duval, P., M. F. Ashby and I. Anderman. 1983. Rate-controlling processes in the creep of polycrystalline ice. F. Phys. Chem., 87(21), 4066-4074.

Gagliardini, O. and J. Meyssonnier. 1999a. Analytical derivations for the behaviour and fabric evolution of a linear orthotropic ice polycrystal. 7. Geophys. Res., 104(B8), 17,797-17,809.

Gagliardini, O. and J. Meyssonnier. 1999b. Plane flow of an ice sheet exhibiting strain-induced anisotropy. In Hutter, K., Y. Wang and H. Beer, eds. Advances in cold-region thermal engineering and sciences: technological, environmental, and climatological impact. Berlin, etc., Springer-Verlag, 171-182. (Lecture Notes in Physics 533.

Gagliardini, O. and J. Meyssonnier. 2000. Simulation of anisotropic ice flow and fabric evolution along the GRIP-GISP2 flowline, central Greenland. Ann. Glaciol., 30, 217-223.

Gödert, G. and K. Hutter. 1998. Induced anisotropy in large ice shields: theory and its homogenization. Continuum Mech. Thermodyn., 10(5), 293-318.

Gödert, G. and K. Hutter. 2000. Material update procedure for planar transient flow of ice with evolving anisotropy. Ann. Glaciol., 30, 107-114.

Gundestrup, N., D. Dahl-Jensen, S. J. Johnsen and A. Rossi. 1993. Bore-hole survey at dome GRIP 1991. Cold Reg. Sci. Technol., 21 (4), 399-402.

Lliboutry, L. 1993. Anisotropic, transversely isotropic nonlinear viscosity of rock ice and rheological parameters inferred from homogenization. Int. 7. Plasticity, 9 (5), 619-632.

Mangeney, A. and F. Califano. 1998. The shallow ice approximation for anisotropic ice: formulation and limits. 7. Geophys. Res., 103(B1), 691-706.

Mangeney, A., F. Califano and O. Castelnau. 1996. Isothermal flow of an anisotropic ice sheet in the vicinity of an ice divide. 7. Geophys. Res., $101(\mathrm{~B} 12)$, 28,189-28,204.

Mangeney, A., F. Califano and K. Hutter. 1997. A numerical study of anisotropic, low Reynolds number, free surface flow for ice sheet modeling. $\mathcal{F}$. Geophys. Res., 102(B10), 22,749-22,764.

Meyssonnier, J. and A. Philip. 1996. A model for the tangent viscous behaviour of anisotropic polar ice. Ann. Glaciol., 23, 253-261.

Philip, A. and J. Meyssonnier. 1999. Anisotropic isothermal ice-cap flow with the shallow ice approximation. In Hutter, K., Y. Wang and H. Beer, eds. Advances in cold-region thermal engineering and sciences: technological, environmental, and climatological impact. Berlin, etc., Springer-Verlag, 237-248. (Lecture Notes in Physics 533.)

Savvin, A., R. Greve, R. Calov, B. Mügge and K. Hutter. 2000. Simulation of the Antarctic ice sheet with a three-dimensional polythermal icesheet model, in support of the EPICA project. II. Nested high-resolution treatment of Dronning Maud Land, Antarctica. Ann. Glaciol., 30, 69-75.

Van der Veen, C. J. and I. M. Whillans. 1994. Development of fabric in ice. Cold Reg. Sci. Technol., 22(2), 171-195.

Vialov, S. S. 1958. Regularities of glacial shields movement and the theory of plastic viscours [sic] flow. International Association of Scientific Hydrology Publication 47 (Symposium at Chamonix 1958 - Physics of the Movement of the Ice), 266-275. 